\title{
ELECTRON MICROSCOPIC STUDY OF THE DEVELOPMENTAL STAGES OF EIMERIA INTESTINALIS CHEISSIN, 1948 IN DOMESTIC RABBIT (ORYCTOLAGUS CUNICULUS) WITH REFERENCE TO ENDODYOGENY \\ By
}

\author{
HODA ELFAYOUMI ${ }^{*}$ AND HEBA M. ABDEL-HALEEM \\ Department of Zoology, Faculty of Science, Beni-Suef University, Beni-Suef, \\ Egypt ( ${ }^{*}$ Corresponding author: h.elfayoumi@yahoo.com)
}

\begin{abstract}
The endogenous stages in Eimeria intestinalis were studied in experimentally infected coccidia-free rabbits by transmission electron microscopy. Four asexual generations were observed.Two types of merozoites were reported. Binucleated merozoites possess all the characteristics of apical complex indicating asexual reproduction by endodyogeny. While the second type, the mononuclear merozoites have one central nucleus. Gamonts were developed mainly from the third generation merozoites where the mature gamonts were recorded together with the fourth generation schizonts. Macro- and microgametogenesis were clearly observed. Development of microgametes and its fine structural characteristics were detected. Mature macrogametes with central large nucleus and the appearance of two types of wall forming body (I, II) were observed. Many reserve food materials including amylopectin granules and lipids droplets were very characteristic. Control experimentally infected rabbits shed unsporulated oocysts on the day eight p.i.
\end{abstract}

Key words: Electron Microscopy, Eimeria Intestinalis developmental Stages, Domestic Rabbit (Oryctolagus Cuniculus).

\section{Introduction}

Coccidia of the genus Eimeria are the most common parasites of the rabbit and are responsible for major economic losses in rabbit farming (Oncel et al, 2011). Among 15 Eimeria species infecting rabbits in different parts of the world, Eimreia intestinalis is undoubtedly one of most pathogenic coocidia species in rabbits and it has considerable immunogenicity (Licois et al, 1992). However, studies of E. intestinalis are scarce and fragmented (Kheysin, 1965, Snigirevskaya, 1969). To the best of the authors' knowledge, some endogenous stages of E. intestinalis life cycle were reported by light microscopy (ElShahawi et al, 2012). Also, electron microscopic studies were performed on merozoite formation (Snigirevskaya, 1969, Licois et al, 1992) and on microgametogenesis (Kheysin 1958). Since then, no one study containing all the stages of $E$. intestinalis life cycle. Therefore, the present study aimed to investigate the all stages of E. intestinalis life cycle by means of light and electron microscopy.

\section{Materials and methods}

Eighteen coccidia-free rabbits were inoculated orally with approximately $1 \times 10^{5}$ sporulated purified oocysts of E. intestinalis isolated from the natural infection in Egypt by single oocyst method (Li and Ooi, 2009, Kvičerová et al, 2008). The faeces of the inoculated animals were examined daily after inoculation. The rabbits used in this study were killed at 24, 48, 72 , $96,120,144,168,192 \& 216$ h postinfection (p.i.). Samples of proximal and distal ileum were removed and prepared for light and electron microscopic studies. They were placed immediately in 3\% glutaraldehyde in a $0.1 \mathrm{M}$ cacodylate buffer (pH 7.3) for $24 \mathrm{~h}$. After postfixation in $2 \%$ $\mathrm{OsO} 4$ for $4 \mathrm{~h}$ dehydration took place in ascending ethanol series. Samples were processed in the usual manner and embedded in an araldite embedding medium. Semi-thin sections were cut with Leica ul- 
tracut UC7 and stained with toluidine blue for light microscopic examination. Ultrathin sections were stained with uranyl acetate and lead citrate and examined with a JEOL-JSM-1011 electron microscope at $80 \mathrm{kV}$. All measurements of different stages are in $\mu \mathrm{m}$.

\section{Results}

Schizogony: A total of four asexual generations were observed, invading sporozoites but were very difficult to be identified. The early developmental stages were transformed to uninuclear schizonts observed at $24 \mathrm{~h}$ p.i. in the epithelial cells along the entire length of the villi. They could identify by their bounding parasitophorous vacuole and by the presence of few remnants of apicomplexan ultrastructural characteristics of the coccidian motile stages such as micronenes, conoid, rhoptries and remnant of the pellicle (Fig. 1). A number of nuclear divisions took place and the produced daughter nuclei migrated to the periphery producing the first generation multinuclear schizonts at $48 \mathrm{~h}$ p.i. (Fig. 2). At $72 \mathrm{~h}$ p.i., mature schizonts of the first generation with about 3-7 fully formed merozoites were observed free within a parasitophorous vacuole. After the first generation schizogeny, $2^{\text {nd }}$ and $3^{\text {rd }}$ generations proceeded in the same ways and detected at 120 and $168 \mathrm{~h}$ p. i. respectively. The only difference in this process was the gradual increase in number of the resulting fully formed merozoites reaching about 25 individual at the end of the $3^{\text {rd }}$ generation (Fig. 3). During the third schizogonic cycle, two types of merozoites could be distinguished (Figs. 4, 5). The first type, binuclear merozoites with two nuclei measuring $4.3(4-5) \mu \mathrm{m} \times 2(1.5-2.5) \mu \mathrm{m}$ (Fig. 4). The second type, mononuclear merozoites with one central nucleus and they measured 5 (4.5-6) $\mu \mathrm{mx} 1.5$ (1.2-2) $\mu \mathrm{m}$ (Fig. 5). Both types of merozoites showed the typical coccidian fine structure including: the pellicle, subpellicular microtubules, conoid, rhoptries, micronemes a well developed endoplasmic reticulum, amylopectin granules and a normally structured nucleus (Figs. 4, 5,6). Mature schizonts of the fourth generation were noted $192 \mathrm{~h}$ p.i. Each schizont containing 6-23 fully formed merozoites. Also, the two types of merozoites still could be seen. The presence of binucleated merozoites indicated the specific multiplication process known endodyogeny in which two daughter merozoites are developed inside the mother cell or the metrocytes which still retained all the characteristics of the apical complex. This process were clearly observed in the last asexual cycle beside normal ectomerogeny process in which uni- and multinuclear schizonts are formed resulting in the formation of large number of developed merozoites.

Microgametogenesis: In the present study, microgametes were observed at 168 h.p.i. The electron microscopic study showed that the positive demarcation of the multinucleated microgamonts was confirmed by the presence of one or two centrioles in vicinity of the peripherally arranged nuclei, cytoplasmic fissure on the surface of the microgamont and the outer most plasma membrane enclosing the gamont (Figs.7, 8). The microgamont undergoes subdivision into several, multinuclear, cytomere-like masses. The differentiation of microgametes began when elevations appeared adjacent to the nuclei and the centrioles. An elongation of the nucleus leads to a finger-like protuberance incorporating one mitochondrion and developed finally into a microgamete. Portions of the microgamont nuclei form the residual nuclei that remained within the residual body of the mother microgamont (Fig. 8). The residual body also contained mitochondria and amylopectin granules. The developing microgametes increased in length and eventually become detached and occupied the parasitophorous vacuole (Fig.9). The fully formed microgamete had an elongated nucleus, tubular mito- 
chondrion and clearly observed two flagella. Nucleus occupied nearly all the space of the microgamete, while a single stretched mitochondrion was located in the nuclear depression and extended along the anterior half of the microgamete nucleus (Fig. 9). Flagella extended posteriorly from the basal bodies and appeared near the anterior end of the microgamete. The exact number of flagella per microgamete was difficult to determine during this study but according to our observation, it seemed to be at least two flagella in each microgamete (Fig.9).

Macrogametogenesis: The electron microscopic study revealed that the developing macrogamont were characterized by a central large nucleus with prominent nucleolus. The most indicative structure was the appearance of the so called wall forming bodies (WFB). Two types of wall forming bodies were detected (WFI, WFII). The wall forming body of the second type appears at first. These were large homogenous, osomophilic inclosing in narrow lacunae arranged at the periphery (Fig 11). The other type of wall forming bodies (WFI) appeared later were smaller dense osmophilic and arranged peripherally in the cytoplasm. These stages were richly supplied with reserve food materials amylopectin granules and lipids droplets were clearly seen inside this stage (Fig11, 12). After fertilization of the oocyst wall was initiated. The first indication of post fertilization changes in the young zygote was the disaggregation and/or fusion of the wall-forming bodies of each type together with remarkable decrease in their numbers while the number of the amylopectin granules was remarkably increased (Fig. 12). The disintegration and the gradual disappearance of the wall forming bodies were correlated with the onset of the appearance of the inner and the outer wall of the oocyst (Fig. 12). After the completion of the oocyst wall formation, the wall forming bodies were no longer observed. Fully formed nonsporulated oocysts were shedded in the faeces of experimentally infected rabbits control on the day eight p.i. These were allowed to sporulated which was completed within $60 \mathrm{~h}$ at $25 \pm 3^{\circ} \mathrm{C}$. The morphology of the sporulated oocysts is shown in figure (13).

\section{Discussion}

The majority of Eimeria species were described based only on their oocyst morphology with no information about the life-cycle and the biology of most species. Although Eimreia intestinalis is one of the most pathogenic Coccidia species in rabbits, the studies on its life cycle are scarce and fragmented (El-Shahawi et al, 2012). Electron microscopy has been performed on merozoite formation (Snigirevskaya, 1969; Licois et al, 1992) and on microgametogenesis (Kheysin, 1958) of E. intestinalis. In the present study, all the life cycle stages were observed and the fine structural characteristics were analyzed applying the transmission EM. Regarding the merogonic cycle, four asexual generations were encountered in the epithelial cells of the small intestine. This agreed with Licois et al. (1992) and El-Shahawi et al. (2012), where four asexual generations for $E$. intestinalis were also recorded. But, three asexual generations only for the same parasite have been reported in other studies (Kheysin, 1958; Pellérdy, 1974; Peeters et al, 1984). The difference in the number of schizogonic generations may be due to the different dose of oocysts used at the beginning of the life cycle (Licois et al, 1992; Pakandl et al, 2003) or other ecological factors which could affect the events of the life cycle. In general, the exact number of asexual generations among the genus Eimeria is not fixed (Dai et al, 2005; Mehlhorn, 2006).

Most interesting, two types of merozoites (mononuclear and binuclear) were observed in the present study which is recorded for the first time for such parasites. 
The presence of binucleated merozoites may indicate the specific multiplication process known endodyogeny as suggested by Scholtyseck 1979). On the other hand, Streun et al. (1979) postulates that the mononuclear merozoites give rise to macrogamonts and hence they are female. Analogously, the multinuclear merozoites should be male and give rise to microgamonts. The amylopectin granules present both in the mononuclear merozoites of the third generation and in the macrogametes might confirm that these merozoites give rise to the macrogamonts. This observation suggested that gamogony might require only three schizogonies and the fourth generation was thought to prolong oocyst production (Licois et al. 1992). This finding could support the role of both types of merozoites in the life cycles of rabbit coccidian that was proposed by Streun et al. (1979).

The ultrastructural architecture of merozoites produced in the four generation was nearly similar. These merozoites had the apicomplexan characteristics including pellicle, conoid, rhoptries, micronemes and subpellicular microtubules (Scholtyseck, 1973; Licois et al, 1992; Pakandl et al, 2003, Jelínková et al, 2008 Mehlhorn and Bunnag, 1988).

In the present study, gamonts were developed mainly from the third generation merozoites where the mature gamonts were recorded together with the fourth generation schizonts. Similar results were reported by Licois et al. (1992) and ElShahawi et al. (2012) in E. intestinalis and Jelínková et al. (2008) in E. exigua infecting rabbit.

The microgametogenesis process occurred in two phases; growth phase and differentiation phase. In the growth phase, mitotic nuclear division occurred and the resulted nuclei later became arranged peripherally. The sequences of these events during the growth phase were similar to that reported for many other species of
Eimeria (Scholtyseck 1979, Mehlhorn and Bunnag 1988). During the present study, the microgamont were subdivided into several, multinuclear, cytomere-like masses. Similar phenomena were shown in $E$. maxima of chickens (Scholtyseck 1973). In the differentiation phase, microgametes developed through karyoplasmic separation of the electron dense from the electron-pale of the nuclear materials which incorporated as the future microgamete nucleus. An elongation of the microgamete nucleus leads to a finger-like protuberance incorporating one mitochondrion with flagellar buds and developed finally into a microgamete. Such observations were recorded for other Eimeria species (Kheysin, 1965, Scholtyseck, 1973, Abdel-Ghaffar et al, 1990, Al-Ghamdy et al, 2005). However, Scholtyseck (1965) found that after repeated nuclear divisions in the microgamont, each resulting nucleus represent the nucleus of the developed during the microgametogenesis of E. perforans. At least two definite flagella were recorded in each mature microgamete. The exact number of flagella found in mature microgametes cannot be detected through the present study. This finding was similar to that in $E$. intestinalis (Kheysin, 1965) and E. magna (Al-Ghamdy et al, 2005). On the other hand, three flagella were recorded in other rabbit Coccidia as those in E. perforans (Scholtyseck, 1965). This variation is interspecific and intraspecific and having significance in the area of phylogeny and systematic (Slapeta et al, 2003; AlGhamdy et al, 2005).

Maturation of the coccidian macrogamete and the formation of oocyst wall are always of great importance. This is because such phase of development culminated in the production of the infective stage which is highly resistant to various environmental factors and initiated the subsequent infection of new hosts (Speer et al, 1973, AlGhamdy et al, 2005). The present study showed that the developing macrogamonts 
had two types of these wall forming body I and wall forming bodies II. Both types of the wall forming bodies were named after their fate of function rather than the order of their appearance. Therefore, the wall forming bodies I that appeared after the wall forming body II was fused earlier and gives rise to the outer layer of the future oocyst wall while the wall forming body II was fused to form the inner layer (AbdelGhaffar et al, 1990, Al-Ghamdy et al, 2005). However, Entzeroth et al. (1981) found that the macrogamonts of E. truncata contained only one type wall forming body. Also, some other apicomlpexan genera as Klossia, Aggregata and Protococcidia found to be not contained these wall forming bodies (Scholtyseck, 1973). Thus, Mehlhorn (2006) concluded that the number of wall forming bodies is a genus dependent character. As the development proceeded, the wall forming body I arranged more peripherally while wall forming body II arranged more centrally and large amount of food reserve (amylopectin granules and lipids) noticed in the cytoplasm of the mature macrogamonts. Similar observations were reported (Dai et al, 2005; Bashtar et al, 2010). After fertilization the wall-forming bodies fused together giving rise to the oocyst wall. Oocysts with a double wall were shed in the feces of the infected host on day 8 p.i. The appearance of the bilayered oocyst wall is usually accompanied with the disappearance of the wall forming bodies in the young oocyst. These results are in agreement with previously reported results in many Eimeria species (Teixeira et al, 2004, Bashtar et al, 2010, El-Shahawi et al, 2012).

\section{References}

Abdel-Ghaffar, F, Marzouk, M, Ashour, MB, Mosaad, MN, 1990: Effects of Eimeria labbeana and E. stiedai infection on the activity of some enzymes in the serum and liver of their hosts. Parasitol. Res. 76:440-3

Al-Ghamdy, AA, Shazly, M, Al-Rasheid, K, Muborak, M, Bashtar, A, 2005: Light and electron microscopy of Eimeria magna Pérard, 1925 infecting the house rabbit, Oryctolagus cuniculus from Saudi Arabia. II. Gamogony and oocyst wall formation. Saudi J. Biol. Sci. 12:114-25

Bashtar, A, Abdel-Ghaffar, F, Al-Rasheid, K, Mehlhorn, H, Al Nasr, I, 2010: Light microscopic study on Eimeria species infecting Japanese quails reared in Saudi Arabian farms. Parasitol. Res. 107:409-16

Dai, Y, Liu, X, Lju, M, Tao, J, 2005: The life cycle and pathogenicity of the coccidium Eimeria nocens (Kotlan, 1933) in domestic goslings. J. Parasitol. 91:1122-5

El-Shahawi, GA, El-Fayomi, HM, AbdelHaleem, HM, 2012: Coccidiosis of domestic rabbit (Oryctolagus cuniculus) in Egypt: light microscopic study. Parasitol. Res. 110:251-8 Jelínková, A, Licois, D, Pakandl, M, 2008: The endogenous development of the rabbit coccidium Eimeria exigua Yakimoff, 1934. Vet. Parasitol. 156:168-72

Kheysin, YM, 1958: Cytologische untersuchungen verschiedener Stadien des Lebenszyklus der Kaninchencoccidien. I. Eimeria intestinalis Cheissin 1948. Arch. Protistenk. 102: 265-89

Kheysin, YM, 1965: Electron microscopic study of microgametogenesis in two species of coccidia from rabbit (Eimeria magna and $E$. intestinalis). Acta Protozool 3: 215-234

Kvičerová, J, Pakandl, M, Hypša, V, 2008: Phylogenetic relationships among Eimeria spp. (Apicomplexa, Eimeriidae) infecting rabbits: evolutionary significance of biological and morphological features. Parasitol. 135:443-52

Li, M, Ooi, H, 2009: Fecal occult blood manifestation of intestinal Eimeria spp. infection in rabbit. Vet. Parasitol. 161:327-9

Licois, D, Coudert, P, Bahagia, S, Rossi, G L, 1992: Endogenous development of Eimeria intestinalis in the rabbit (Oryctolagus cuniculus). J. Parasitol. 78:1041-8

Mehlhorn, H, 2006: Morphology. In: Parasitology in Focus, Facts and Trends. 3rd Ed., Springer, Berlin

Mehlhorn, H, Bunnag, D, 1988: Parasitology in focus: Facts and trends. Springer-Verlag: Berlin and New York.

Oncel, T, Gulegen, E, Senlik, B, Bakirci, S, 2011: Intestinal coccidiosis in angora rabbits (Oryctolagus cuniculus) caused by Eimeria 
intestinalis, Eimeria perforans and Eimeria coecicola. YYU Vet. Fak. Derg. 22:27-9

Pakandl, M, 1988: Description of Eimeria vejdovskyi sp. n. and redescription of Eimeria media Kessel, 1929. Folia Parasitol. 35:1-9

Pakandl, M, Ĉerník, F, Coudert, P, 2003: The rabbit coccidium Eimeria flavescens Marotel and Guilhon, 1941: an electron microscopic study of its life cycle. Parasitol. Res. 91:304-11

Peeters J E, Charlier G, Antoine O, Mammerickx M (1984) Clinical and pathological changes after Eimeria intestinalis infection in rabbits. Zentralbl Veterinarmed B 31: 9-24

Pellérdy, L, 1974: Coccidia and Coccidiosis. $2^{\text {nd }}$ ed. Paul Parey, Berlin, Hamburg, Germany. Scholtyseck, E, 1965: Die Mikrogametenentwicklung von Eimeria perforans. Z. Zellforsch. 66:625-42

Scholtyseck, E, 1973: Ultrastructure. In: The Coccidia: Eimeria, Isospora, Toxoplasma, and Related Genera. Hammond, DM, Long, PL, (ed.); University Park Press, Baltimore and Butterworth, London.

Scholtyseck, E, 1979: The fine structure of parasitic Protozoa. Berlin, New York: Springer-Verlag

\section{Legends}

Figs. (1-13): Electron micrographs of four generations of schizonts of E. intestinalis in intestinal epithelia of experimentally infecting rabbits (O. cuniculus).

Fig. 1: Unicellular schizont with large central nucleus in parasitophorous vacuole (PV) during the transformation process, where some apicomplexan structures conoid (C), rhoptries $(\mathrm{Rh})$, few micronemes (white arrows head) and pellicle remnants $(\mathrm{PE})$ of the sporozoite structure persist, 24h p.i. Scale bar $=1 \mu \mathrm{m}$.

Fig. 2: Multinuclear schizont (Sch) of first generation with many nuclei (N) arranged at periphery and enclosed by parasitophorous vacuole (PV), $48 \mathrm{~h}$ p.i. Scale bar $=2 \mu \mathrm{m}$.

Fig. 3: Mature shizonts (Sch) of first generation with fully formed merozoites (Me) within a parasitophorous vacuole (PV). Merozoites (Me) with rhoptries $(\mathrm{Rh})$, nucleus $(\mathrm{N})$ and nucleolus $(\mathrm{Nu}), 72 \mathrm{~h}$ p.i. Scale bar $=2 \mu \mathrm{m}$.

Fig. 4: Binuclear merozoite with apicomlexan characteristics and two nuclei $(\mathrm{N})$.

Fig. 5: Mononuclear merozoite. Note: the presence of only one nucleus $(\mathrm{N})$.

Fig 6: Fourth generation schizonts ( $\mathrm{Sch}$ ) with fully formed merozoites (Me). Merozoites showed a typical coccidian fine structure including: a membranous pellicle $(\mathrm{Pe})$, conoid $(\mathrm{C})$, rhoptries $(\mathrm{Rh})$, micronemes $(\mathrm{Mn})$, amylopectin granules (A), a normally structured nucleus $(\mathrm{N})$ and nucleolus $(\mathrm{Nu}), 192 \mathrm{~h}$ p.i. Scale bar $=2 \mu \mathrm{m}$.

Figs. 7, 8: Early microgamonts (Mi) with peripherally arranged nuclei $(\mathrm{N})$ and centrioles (arrows) appear in vicinity of it. Micropore (MP), cytoplasmic fissure (arrowheads) and electron lucent parasitophorous vacuole (PV) clearly seen. Note: microgamonts subdivided into several multinuclear cytomere-like masses.

Scale bar $=2 \mu \mathrm{m}$.

Figs. 9: Microgametes filling entire parasitophorous vacuole (PV). Each with large nucleus (N), Left and right flagella (F) and one mitochondrion (MI). Scale bar $=1 \mu \mathrm{m}$.

Figs. 10: Early macrogamonts with nucleus $(\mathrm{N})$, nucleolus $(\mathrm{Nu})$, wall forming body II (WFBII) and amylopectin granules (A). Macrogamont enclosed by parasitophorous vacuole (PV). Scale bar $=2 \mu \mathrm{m}$.

Fig. 11: Mature macrogamete with an increase of cytoplasmic inclusions. Note: presence of both types of twall forming bodies (WFBI, WFBII), amylopectin granules (A) and lipids (L). Scale bar $=2 \mu \mathrm{m}$.

Fig. 12: Post-fertlized macrogamete with formation of outer and inner layers of oocyst wall (OL, IL), disaggregating and or fusion of wall forming bodies (WFBII) and great increase of amylopectin granules (A). Scale bar $=2$ $\mu \mathrm{m}$.

Fig. 13: Sporulated oocyst of E. intestinalis. Scale bar $=5 \mu \mathrm{m}$. 


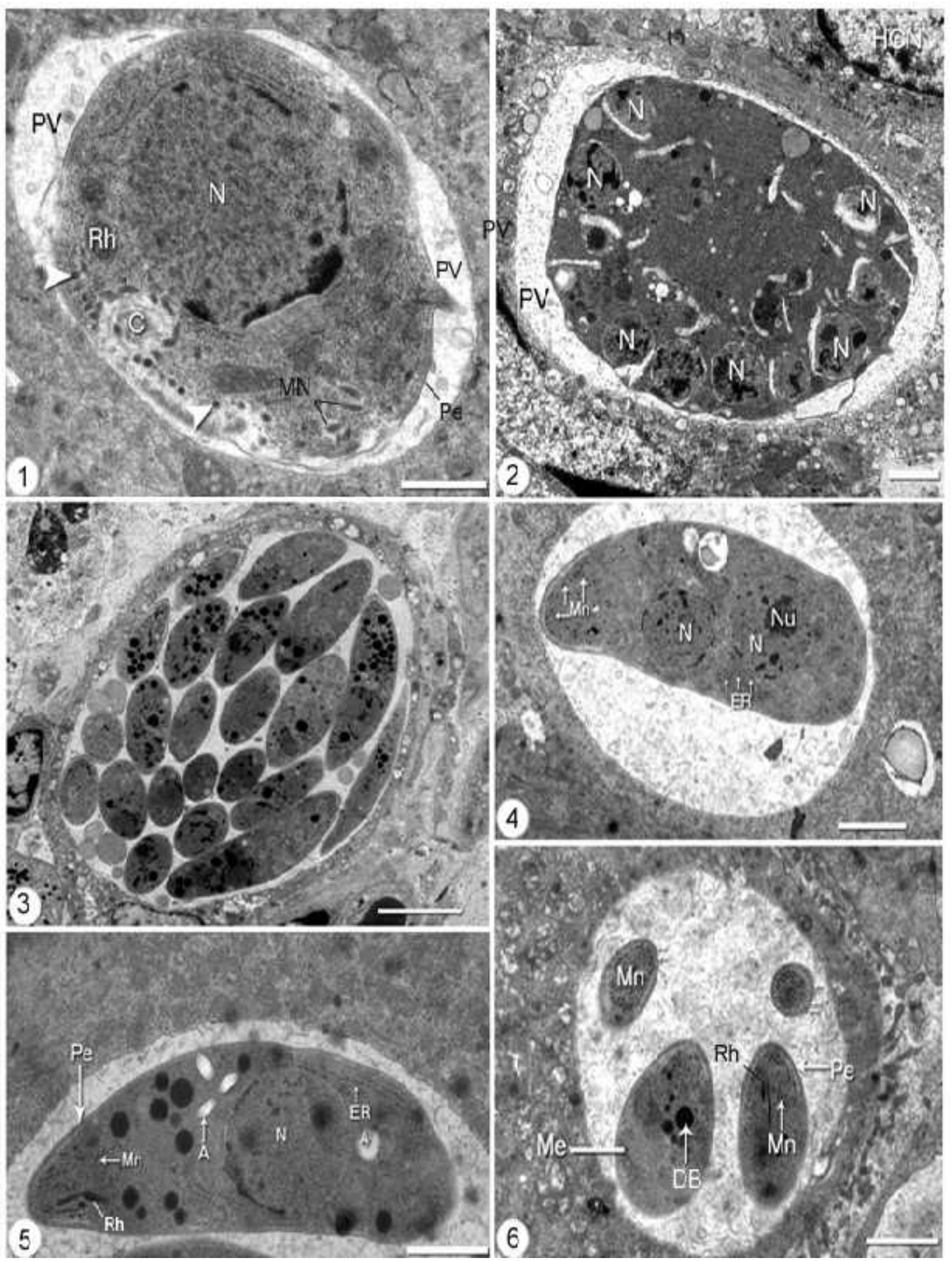



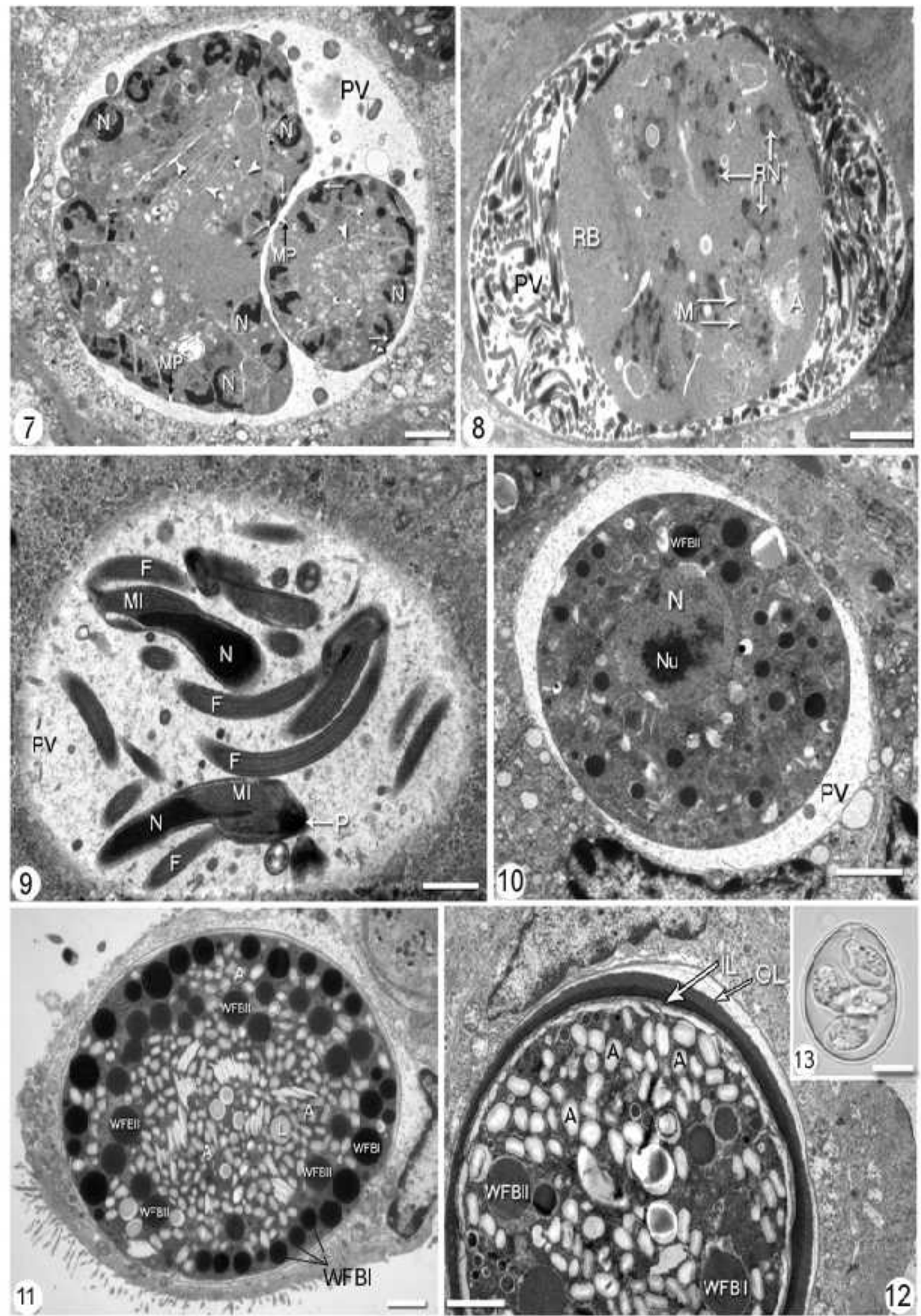